\title{
A Study on the Efficacy and Safety of Rivaroxaban in Urologic Cancer-Associated Venous Thromboembolism
}

\author{
Jang Ho Lee ${ }^{1}$, Dalsan You', Sang-Do Lee ${ }^{1}$, Yeon-Mok Oh${ }^{1}$, Jae Seung Lee ${ }^{1}$ \\ ${ }^{1}$ Department of Pulmonary and Critical Care Medicine, Asan Medical Center, University of Ulsan College \\ of Medicine, Seoul, Korea \\ ${ }^{2}$ Department of Urology, Asan Medical Institute of Convergence Science and Technology, Asan Medical \\ Center, University of Ulsan College of Medicine, Seoul, Korea
}

\begin{abstract}
Purpose: Although direct oral anticoagulants (DOACs) are recommended as an alternative to low-molecular weighted heparin for cancer-associated venous thromboembolism (VTE), there is no firm evidence on the efficacy and safety of DOACs in patients with urologic cancer. Therefore, we compared the efficacy and safety of rivaroxaban and dalteparin for treating cancer-associated VTE in urologic cancer.

Materials and Methods: We reviewed the medical records of 124 eligible VTE patients with urologic cancers who were treated with dalteparin or rivaroxaban. The primary outcome was the composite event of clinically relevant bleeding or VTE recurrence. The secondary outcomes were VTE recurrence, clinically relevant bleeding events, and all-cause mortality.

Results: During anticoagulation period, there was no significant difference in primary and secondary outcomes between the groups. In Cox proportional hazards model for composite events, although there was no statistical significance, rivaroxaban presented lower hazard ratio (HR) than dalteparin $(H R, 0.472 ; 95 \%$ confidence interval [Cl], $0.210-1.060 ; p=0.069$ in univariate analysis; $H R, 0.505 ; 95 \% \mathrm{Cl}, 0.206-1.234 ; p=0.134$ in multivariate analysis). In clinically relevant bleeding events, there was no significance difference between rivaroxaban and dalteparin $(\mathrm{HR}, 0.568 ; 95 \% \mathrm{Cl}, 0.238-1.358 ; \mathrm{p}=0.203$ in univariate analysis; $\mathrm{HR}, 0.617 ; 95 \% \mathrm{Cl}, 0.232-1.636$; $\mathrm{p}=0.331$ in multivariate analysis).

Conclusions: Rivaroxaban can be regarded as a valuable option for VTE in urologic cancer. Further prospective studies are warranted to prove the safety or efficacy of rivaroxaban for treating VTE in patients with urologic cancer. (Korean J Urol Oncol 2019;17:178-185)
\end{abstract}

Key Words: Anticoagulants $\cdot$ Dalteparin $\cdot$ Rivaroxaban $\cdot$ Urologic neoplasm $\cdot$ Venous thromboembolism

Received October 14, 2019, Revised November 15, 2019 ,

Accepted November 25, 2019

Corresponding Author: Jae Seung Lee

Department of Pulmonary and Critical Care Medicine, Center for Pulmonary Hypertension and Venous Thrombosis, Asan Medical Center, University of Ulsan College of Medicine, 88 Olympic-ro 43-gil, Songpa-gu, Seoul 05505, Korea

E-mail: jsdoc1186@hotmail.com

Tel: +82-2-3010-3994, Fax: +82-2-3010-6968

ORCID: https://orcid.org/0000-0003-4130-1486

- This study was supported by University of Ulsan College of Medicine.

\section{INTRODUCTION}

Cancer-associated venous thromboembolism (VTE) is one of the most common and fatal complications of malignancy. ${ }^{1,2}$ The incidence of VTE is relatively low in urologic cancer compared with other cancer types. ${ }^{3-5}$ However, the adequate management of cancer-associated VTE is important to prevent patient mortality. Anticoagulation is the mainstay of VTE treatment in which anticoagulants are ap-

(i) (8) This is an Open Access article distributed under the terms of the Creative Commons Attribution Non-Commercial License (http://creativecommons.org/licenses/by-nc/4.0/) which permits unrestricted non-commercial use, distribution, and reproduction in any medium, provided the original work is properly cited. 2019 (C) Copyright The Korean Urological Oncology Society and The Korean Prostate Society. All Rights Reserved. 
propriately decided by the clinicians to prevent bleeding events in patients. After CLOT trial, low-molecular-weight heparin (LMWH) is regarded as the standard therapy for cancer-associated VTE. ${ }^{6}$ Ascribed to its convenient use, some clinicians prefer to prescribe direct oral anticoagulants (DOACs) for treating cancer-associated VTE. ${ }^{7}$

There are following 2 types of DOACs: direct factor Xa (rivaroxaban, edoxaban, and apixaban) and direct thrombin inhibitor (dabigatran). ${ }^{8}$ Currents guidelines for the treatment of VTE have listed DOACs as one of the standard therapies in the general population. ${ }^{9}$ However, there is no firm evidence supporting the use of DOACs in patients with cancer. ${ }^{10}$ In addition, studies have reported that DOACs increase gastrointestinal and genitourinary tract bleeding compared with other anticoagulants. ${ }^{11-14}$

There are 2 randomized controlled trials (RCTs) suggesting DOACs as reasonable alternatives to $\mathrm{LMWH}^{2,15-18}$ There were no differences in VTE recurrence and major bleeding between DOACs and LMWH, but clinically relevant nonmajor bleeding events increased in the DOACs group. There were several studies that clinically relevant nonmajor bleeding events were increased due to included patients with gastrointestinal cancer. ${ }^{19,20}$ Clinically relevant nonmajor bleeding is one of the deciding factors for the discontinuation or decreasing doses of anticoagulants. In addition, urologic cancer occupied only about $10 \%$ in all patients. Therefore, conclusions of both studies cannot be equally applied to all patients with urologic cancer. Because visceral malignancies with mucosal lesion could increase bleeding risk in patients with cancer, studies on the safety and efficacy of DOACs for treating urologic cancer are necessary. $^{21}$

This study aimed to compare the efficacy and safety of rivaroxaban and dalteparin for the treatment of cancer-associated VTE in patients with urologic cancer.

\section{MATERIALS AND METHODS}

\section{Study Design and Patients}

This was a single-center retrospective study at Asan Medical Center (a 2,700-bed referral hospital in Seoul, Korea). From the electronic medical record system, patients with history of rivaroxaban or dalteparin treatment for urologic cancer were selected. Urologic cancer included blad- der cancer, prostate cancer, kidney cancer, testicular cancer, and other urinary tract cancers. There were 3 inclusion criteria in this study: (1) diagnosis of primary urologic cancer, (2) diagnosis of pulmonary embolism (PE) and/or deep vein thrombosis (DVT) and (3) treatment of VTE with rivaroxaban or dalteparin between 1 January 2012 and 31 December 2017. Exclusion criteria were (1) prescription of rivaroxaban or dalteparin for 7 days or less without bleeding events; (2) no follow-up visits after the first visit; (3) rivaroxaban or dalteparin firstly prescribed at other institutions; (4) VTE lesions removed by thromboembolectomy; and (5) changing of the anticoagulant dose during therapeutic periods.

Because there was no recommendation for the treatment of cancer-associated VTE in Asan Medical Center, anticoagulant prescription was decided on clinical judgement made by responsible clinicians considering patients' condition, preference of clinicians or patients, comorbidity and so on. In rivaroxaban group, cancer-associated VTE is treated with oral rivaroxaban $15 \mathrm{mg}$ twice daily for 21 days followed by $20 \mathrm{mg}$ once daily. In dalteparin group, patients subcutaneously injected dalteparin $200 \mathrm{IU} / \mathrm{kg}$ once daily.

This study was approved by the Institutional Review Board of the Asan Medical Center (IRB no. 2017-0652). The requirement for informed consent was waived due to the retrospective nature of this study.

\section{Measurement}

We collected the patient clinical characteristics and study outcomes through retrospective review of their electronic medical records. Additionally, we calculated the score of bleeding risk factors. Bleeding risk factors included surgical history 2 weeks before anticoagulant use, concurrent use of antiplatelet agents, accompaniment of a primary or metastatic brain tumor, regionally advanced or metastatic cancer, coexisting gastrointestinal or urothelial cancer, and bevacizumab use in 6-week period before anticoagulant use. ${ }^{17}$

VTE included PE and/or DVT. PE was diagnosed by certificated radiologists via computed tomography or ventilation-perfusion scanning. Doppler ultrasonography or computed tomography venography was used for the diagnosis of DVT. Follow-up examinations were performed when clinicians suspected recurrence of VTE or prescribed imaging study for regular evaluation of cancer status. 
The composite event, including any events of major bleeding, clinically relevant nonmajor bleeding events or recurrence events during anticoagulant therapy, was selected as the primary outcome in this study. We defined VTE recurrence as computed tomographic or ultrasonographic evidence of an increased extent or new occurrence of VTE during anticoagulant therapy. ${ }^{17}$ The evaluation of VTE recurrence was performed when VTE recurrence was suspected or periodical examination to assess the cancer status. Clinically relevant bleeding events were regarded as major bleeding events during anticoagulant therapy when one of the following criteria was met: (1) death-related, (2) VTE occurrence at a fatal site (intracranial, intraocular, retroperitoneal, intraspinal or pericardial) and (3) required a transfusion of at least 2 units of packed red blood cells or a hemoglobin decrease of at least $2.0 \mathrm{~g} / \mathrm{dL}^{22}$ Any other clinically relevant events which were not included in the major bleeding events were regarded as clinically relevant nonmajor bleeding. Secondary outcomes included VTE recurrence rate, bleeding event during anticoagulant therapy and death until 31 March 2019.

For subgroup analysis, we defined that urothelial cancer included bladder cancer, kidney cancer and other urinary tract cancer. Other urinary tract cancer included ureter cancer and urethral cancer.

\section{Statistical Analysis}

Differences in categorical variables were analyzed by

Table 1. Baseline characteristics of the study cohort

\begin{tabular}{|c|c|c|c|}
\hline Characteristic & Dalteparin $(n=61)$ & Rivaroxaban $(\mathrm{n}=63)$ & p-value \\
\hline Male sex & 48 (78.7) & $55(87.3)$ & 0.201 \\
\hline Age & $68.74 \pm 9.58$ & $68.05 \pm 11.17$ & 0.713 \\
\hline Smoking & & & 0.304 \\
\hline Current & $11(18.0)$ & $6(9.5)$ & \\
\hline Ex-smoker & $19(31.1)$ & $18(28.6)$ & \\
\hline $\mathrm{GFR}<50 \mathrm{~mL} / \mathrm{min} / 1.73 \mathrm{~m}^{2}$ & $7(11.5)$ & $6(9.5)$ & 0.723 \\
\hline Platelet count $<100,000 / \mu \mathrm{L}^{*}$ & $2(3.3)$ & $2(3.2)$ & $>0.999$ \\
\hline Cancer type & & & 0.795 \\
\hline Bladder cancer & $25(41.0)$ & $20(31.7)$ & \\
\hline Prostate cancer & $13(21.3)$ & $19(30.2)$ & \\
\hline Kidney cancer & $16(26.2)$ & $17(27.0)$ & \\
\hline Testicular cancer & $1(1.6)$ & $1(1.6)$ & \\
\hline Other urinary tract cancer & $6(9.8)$ & $6(9.5)$ & \\
\hline Metastasis & $49(80.3)$ & $37(58.7)$ & 0.009 \\
\hline Coexisting cancer* & $8(13.1)$ & $2(3.2)$ & 0.052 \\
\hline Brain lesion* & $4(6.6)$ & $3(4.8)$ & 0.715 \\
\hline History of chemotherapy & $47(77.0)$ & $36(57.1)$ & 0.018 \\
\hline History of radiotherapy & $12(19.7)$ & $12(19.0)$ & 0.930 \\
\hline Pulmonary embolism & $39(63.9)$ & $37(58.7)$ & 0.552 \\
\hline History of VTE* & $0(0)$ & $4(6.3)$ & 0.119 \\
\hline Recent operation* & $1(1.6)$ & $6(9.5)$ & 0.115 \\
\hline IVC filter insertion & $8(13.1)$ & $13(20.6)$ & 0.264 \\
\hline Antiplatelet agent* & $4(6.6)$ & $8(12.7)$ & 0.248 \\
\hline Therapeutic duration & $66.78 \pm 58.49$ & $120.57 \pm 96.14$ & $<0.001$ \\
\hline Risk factors for bleeding & & & 0.015 \\
\hline 0 & $8(13.1)$ & $15(23.8)$ & \\
\hline 1 & $41(67.2)$ & $26(41.3)$ & \\
\hline $2-3$ & $12(19.7)$ & $22(34.9)$ & \\
\hline
\end{tabular}

Values are presented as number (\%) or mean \pm standard deviation.

GFR: glomerular filtration rate, VTE: venous thromboembolism, IVC: inferior vena cava.

Difference between both groups was analyzed by the chi-square test, Fisher exact test, or independent 2-sample t-test.

*Fisher exact test. 
chi-square test or Fisher exact test. Independent 2-sample t-test was used to analyze the differences in continuous variables. The time to composite event or clinically relevant bleeding event was analyzed using the Cox proportional hazard model. Time to event curves was calculated by Kaplan-Meier curves with a log-rank test. Multivariate analyses were performed to adjust the differences in clinical and demographic characteristics between dalteparin and rivaroxaban groups. History of chemotherapy, coexisting cancer and score of bleeding risk factors were included as covariates for multivariate analysis, because they were statistically different between both study groups $(\mathrm{p}<0.1)$.

Statistical significance was set at $\mathrm{p}$-value $<0.05$. We used IBM SPSS Statistics ver. 21.0 (IBM Co., Armonk, NY, USA) analytic software for statistical analysis.

\section{RESULTS}

A total of 151 patients with urologic cancer were diagnosed with PE and/or DVT between January 1, 2012 and December 31, 2017. Total 27 patients were excluded from the study, 12 were prescribed with rivaroxaban or dalteparin for 7 days or less without bleeding events, 7 were transferred to other hospitals without visiting our institute, 5 were firstly prescribed at other institutions, dose of anticoagulants was changed during anticoagulant therapy in 2 , and VTE lesions in 1 were removed by pulmonary thromboembolectomy. Among 124 patients, 61 were included in dalteparin group and 63 in rivaroxaban group.

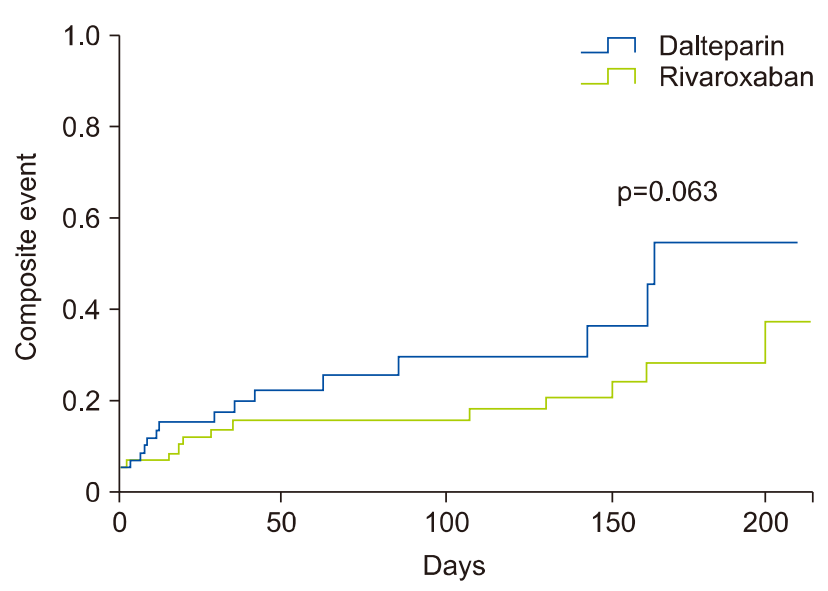

Fig. 1. Kaplan-Meier cumulative event rates for the composite event during 200 days.
Table 1 presents the baseline characteristics of patients in dalteparin and rivaroxaban groups. Patients in the dalteparin group had metastasis and history of chemotherapy, whereas those in the rivaroxaban group underwent respective anticoagulant therapy longer than that in dalteparin group. Patients with one bleeding risk factor were included in dalteparin group, other patients more included in rivaroxaban group.

There was no statistical difference in the composite event between the 2 groups during anticoagulant therapy (dalteparin vs. rivaroxaban group $=23.0 \%$ vs. $22.2 \%, \mathrm{p}=0.923$ ). Kaplan-Meier curve with a log-rank test for time to composite event presented no statistical significance between the 2 groups ( $\mathrm{p}=0.063$ ) (Fig. 1). Other end points, such as bleeding events and mortality, did not present statistical difference between both groups (Table 2). We performed subgroup analyses for urothelial cancer and nonurothelial cancer (Tables 3,4). There were no significant differences in primary and secondary outcomes between rivaroxaban and dalteparin in both subgroup analyses.

In the univariate analysis by Cox proportional hazards model for composite events, rivaroxaban presented 0.472fold hazard ratio (HR) without significance $(95 \%$ confidence interval $[\mathrm{CI}], 0.210-0.160, \mathrm{p}=0.069$ ) (Table 5). After adjusted for history of chemotherapy, coexisting cancer and bleeding risk factors, rivaroxaban did not present statistical significance difference in composite events (HR, 0.505;

Table 2. Primary and secondary endpoints in both groups

\begin{tabular}{lrrr}
\hline \multicolumn{1}{c}{ Variable } & $\begin{array}{c}\text { Dalteparin } \\
(\mathrm{n}=47)\end{array}$ & $\begin{array}{c}\text { Rivaroxaban } \\
(\mathrm{n}=43)\end{array}$ & p-value \\
\hline Composite event & $14(23.0)$ & $14(22.2)$ & 0.923 \\
Recurrence* & $3(4.9)$ & $2(3.2)$ & 0.677 \\
$\begin{array}{l}\text { Symptomatic } \\
\text { recurrence* }\end{array}$ & $1(1.6)$ & $1(1.6)$ & $>0.999$ \\
Bleeding & $11(18.0)$ & $13(20.6)$ & 0.714 \\
Major bleeding* & $4(6.6)$ & $3(4.8)$ & 0.715 \\
CRNM bleeding & $7(11.5)$ & $10(15.9)$ & 0.477 \\
All-cause mortality & $44(72.1)$ & $41(65.1)$ & 0.398 \\
$\begin{array}{l}\text { Bleeding-related } \\
\text { mortality* }\end{array}$ & $3(4.9)$ & $0(0)$ & 0.116 \\
\hline
\end{tabular}

Values are presented as number (\%).

CRNM: clinically relevant nonmajor.

Difference between both groups was analyzed by the chisquare test or Fisher exact test.

*Fisher exact test. 
Table 3. Primary and secondary endpoints in both groups with urothelial cancer

\begin{tabular}{lrrr}
\hline \multicolumn{1}{c}{ Variable } & $\begin{array}{c}\text { Dalteparin } \\
(\mathrm{n}=61)\end{array}$ & $\begin{array}{c}\text { Rivaroxaban } \\
(\mathrm{n}=63)\end{array}$ & p-value \\
\hline Composite event & $11(23.4)$ & $11(25.6)$ & 0.810 \\
Recurrence* & $3(6.4)$ & $1(2.3)$ & 0.618 \\
$\begin{array}{l}\text { Symptomatic } \\
\text { recurrence* }\end{array}$ & $1(2.1)$ & $1(2.3)$ & $>0.999$ \\
Bleeding & $8(17.0)$ & $10(23.3)$ & 0.460 \\
Major bleeding* & $4(8.5)$ & $2(4.7)$ & 0.679 \\
CRNM bleeding & $4(8.5)$ & $8(18.6)$ & 0.159 \\
All-cause mortality & $35(74.5)$ & $29(67.4)$ & 0.463 \\
$\begin{array}{c}\text { Bleeding-related } \\
\text { mortality* }\end{array}$ & $3(6.4)$ & $0(0)$ & 0.243 \\
\hline
\end{tabular}

Values are presented as number (\%).

CRNM: clinically relevant nonmajor.

Difference between both groups was analyzed by the chisquare test or Fisher exact test.

*Fisher exact test.
Table 4. Primary and secondary endpoints in both groups with nonurothelial cancer

\begin{tabular}{lllr}
\hline \multicolumn{1}{c}{ Variable } & $\begin{array}{c}\text { Dalteparin } \\
(\mathrm{n}=14)\end{array}$ & $\begin{array}{c}\text { Rivaroxaban } \\
(\mathrm{n}=20)\end{array}$ & p-value \\
\hline $\begin{array}{l}\text { Composite event* } \\
\text { Recurrence* }\end{array}$ & $3(21.4)$ & $3(15.0)$ & 0.672 \\
$\begin{array}{l}\text { Symptomatic } \\
\text { recurrence* }\end{array}$ & $0(0)$ & $0(0)$ & $>0.999$ \\
Bleeding* & $3(21.4)$ & $3(15.0)$ & 0.672 \\
Major bleeding* & $0(0)$ & $1(5.0)$ & $>0.999$ \\
CRNM bleeding* & $3(21.4)$ & $2(10.0)$ & 0.627 \\
All-cause mortality & $9(64.3)$ & $12(60.0)$ & 0.800 \\
Bleeding-related & $0(0)$ & $0(0)$ & $>0.999$ \\
mortality* & & & \\
\hline
\end{tabular}

Values are presented as number (\%).

CRNM: clinically relevant nonmajor.

Difference between both groups was analyzed by the chisquare test or Fisher exact test.

*Fisher exact test.

Table 5. Hazard ratio for the composite event in the Cox proportional hazards model

\begin{tabular}{|c|c|c|c|c|}
\hline \multirow{2}{*}{ Covariate } & \multicolumn{2}{|c|}{ Univariate analysis } & \multicolumn{2}{|c|}{ Multivariate analysis } \\
\hline & HR $(95 \%$ CI) & p-value & HR $(95 \%$ CI) & $\mathrm{p}$-value \\
\hline \multicolumn{5}{|l|}{ Anticoagulants } \\
\hline Dalteparin & 1 & & 1 & \\
\hline Rivaroxaban & $0.472(0.210-1.060)$ & 0.069 & $0.505(0.206-1.234)$ & 0.134 \\
\hline History of CTx & $1.465(0.583-3.684)$ & 0.417 & $1.284(0.490-3.366)$ & 0.611 \\
\hline Coexisting cancer & $1.104(0.259-4.709)$ & 0.893 & $0.703(0.157-3.147)$ & 0.645 \\
\hline \multicolumn{5}{|l|}{ Bleeding risk factor } \\
\hline 0 & 1 & & 1 & \\
\hline 1 & $1.645(0.601-4.500)$ & 0.332 & $1.414(0.493-4.047)$ & 0.518 \\
\hline $2-3$ & $1.320(0.396-4.405)$ & 0.652 & $1.376(0.339-4.287)$ & 0.607 \\
\hline
\end{tabular}

HR: hazard ratio, CI: confidence interval, CTx: chemotherapy.

Table 6. Hazard ratio for the clinically relevant bleeding event in the Cox proportional hazards model

\begin{tabular}{|c|c|c|c|c|}
\hline \multirow{2}{*}{ Covariate } & \multicolumn{2}{|c|}{ Univariate analysis } & \multicolumn{2}{|c|}{ Multivariate analysis } \\
\hline & HR $(95 \%$ CI $)$ & p-value & HR $(95 \%$ CI $)$ & p-value \\
\hline \multicolumn{5}{|l|}{ Anticoagulants } \\
\hline Dalteparin & 1 & & 1 & \\
\hline Rivaroxaban & $0.568(0.238-1.358)$ & 0.203 & $0.617(0.232-1.636)$ & 0.331 \\
\hline History of CTx & $1.482(0.542-4.053)$ & 0.443 & $1.307\left(0.456^{-3.748)}\right.$ & 0.619 \\
\hline Coexisting cancer & $1.318(0.306-5.674)$ & 0.711 & $0.938(0.203-4.346)$ & 0.935 \\
\hline \multicolumn{5}{|c|}{ Bleeding risk factor } \\
\hline 0 & 1 & & 1 & \\
\hline 1 & $1.272(0.456-3.547)$ & 0.646 & $1.127(0.383-3.312)$ & 0.828 \\
\hline $2-3$ & $1.041(0.298-3.644)$ & 0.949 & $1.072(0.303-3.800)$ & 0.914 \\
\hline
\end{tabular}

HR: hazard ratio, CI: confidence interval, CTx: chemotherapy. 
Table 7. Bleeding site in both groups

\begin{tabular}{lrll}
\hline \multicolumn{1}{c}{ Site } & $\begin{array}{c}\text { Total } \\
(\mathrm{n}=24)\end{array}$ & $\begin{array}{c}\text { Dalteparin } \\
(\mathrm{n}=11)\end{array}$ & $\begin{array}{c}\text { Rivaro- } \\
\text { xaban } \\
(\mathrm{n}=13)\end{array}$ \\
\hline GI tract bleeding & $6(25.0)$ & $2(18.2)$ & $4(30.8)$ \\
Hematuria & $10(41.7)$ & $3(27.3)$ & $7(53.8)$ \\
Intramuscular bleeding & $1(4.2)$ & $1(9.1)$ & $0(0)$ \\
Petechiae & $1(4.2)$ & $1(9.1)$ & $0(0)$ \\
Epistaxis & $1(4.2)$ & $0(0)$ & $1(7.7)$ \\
Intracranial hemorrhage & $1(4.2)$ & $1(9.1)$ & $0(0)$ \\
Vaginal bleeding & $1(4.2)$ & $0(0)$ & $1(7.7)$ \\
Injection site bleeding & $1(4.2)$ & $1(9.1)$ & $0(0)$ \\
Hemoperitoneum & $1(4.2)$ & $1(9.1)$ & $0(0)$ \\
Operation site bleeding & $1(4.2)$ & $1(9.1)$ & $0(0)$ \\
\hline
\end{tabular}

Values are presented as number (\%).

GI: gastrointestinal.

95\% CI, $0.206-1.234 ; \mathrm{p}=0.134)$. In addition, history of chemotherapy, coexisting cancer, and bleeding risk factors were not associated with the incidence of composite events.

Rivaroxaban presented 0.568 -fold HR for clinically relevant bleeding without statistical significance in univariate analysis (95\% CI, 0.238-1.358; $\mathrm{p}=0.203$ ) (Table 6). After adjusting for covariates in multivariate analysis, there was no statistical significance between the 2 groups (HR, 0.617; 95\% CI, 0.232-1.636; $\mathrm{p}=0.331$ ). Other covariates were not associated with the incidence of clinically relevant bleeding events in univariate and multivariate analyses.

Table 7 presents the bleeding sites in both groups. In dalteparin and rivaroxaban groups, urinary tract was the most common bleeding site $(27.3 \%$ and $53.8 \%)$, followed by gastrointestinal tract bleeding (18.2\% and 30.8\%), respectively.

\section{DISCUSSION}

From the previous 2 RCTs that compared DOACs and LMWH for the treatment of cancer-associated VTE, ${ }^{17,18}$ patients with urologic cancer occupied only $10 \%$ of the study group, and the results shown could not be applied to patients with urologic cancer. We previously performed retrospective studies of the bleeding risk of rivaroxaban in patient with gastrointestinal, lung and gynecologic cancer. ${ }^{23-25}$ In these studies, rivaroxaban was associated with higher incidence of bleeding in treatment of gastrointestinal cancer, but not in lung cancer. These results imply that the efficacy and safety of DOACs are needed to be evaluated in each cancer type. Therefore, this study compared the efficacy and safety of rivaroxaban and dalteparin for the treatment of urologic cancer-associated VTE. To the best of our knowledge, this is the first study to compare DOACs with LMWH in urologic cancer patients with VTE. In our study, rivaroxaban and dalteparin did not present statistical difference in composite events, recurrence, bleeding, and mortality. Because rivaroxaban results in higher bleeding tendency compared with other DOACs, the other types of DOACs could be used for the treatment of urologic cancer-associated VTE. $^{8}$

Ascribed to the different therapeutic duration between dalteparin and rivaroxaban groups, we used Cox proportional hazards model to compare time to composite events and clinically relevant bleeding events. Univariate analysis revealed no significant difference between the 2 groups. Because there were significant differences in metastatic status, duration of anticoagulant therapy, and risk factors for bleeding between the 2 groups, we performed multivariate analysis. After adjusting for covariates, there was no significant difference between the groups. Although there was no statistical significance, rivaroxaban presented less HR compared with dalteparin. Analysis for clinically relevant bleeding events also presented similar results. These results suggest the noninferiority of DOACs compared with LMWH in the treatment of urologic cancer.

In both groups, urinary tract was the most common bleeding site, followed by gastrointestinal tract. There were $45.5 \%$ and $84.6 \%$ urinary tract and gastrointestinal bleeding cases in dalteparin and rivaroxaban groups, respectively. There are several explanations for the urinary tract and gastrointestinal bleeding observed. First, DOACs were alleged to increase gastrointestinal tract bleeding compared with other anticoagulants. ${ }^{19,26,27}$ This might be due to the activity of unabsorbed DOACs in gastrointestinal tract. ${ }^{28,29}$ Second, rivaroxaban is mainly excreted through the urinary tract rather than feces. ${ }^{28}$ Compared with dalteparin, the higher anticoagulants activity of rivaroxaban was observed in the urine. ${ }^{30}$ We speculate that these factors cause higher incidence of gastrointestinal and urinary tract bleeding in rivaroxaban compared with dalteparin. In addition, urinary tract and gastrointestinal bleeding have been observed in other cancer types. ${ }^{18,23,24}$ Thus, it possible that DOACs have 
more effect on the gastrointestinal and urinary tract than LMWH in inducing bleeding, and other sites are less affected by DOACs than LMWH. Although we performed subgroup analysis to prove this hypothesis, there was no statistical difference. Because small population was included in this study, this assumption needs to be evaluated by further studies, especially for urinary tract bleeding.

There are several limitations of the study. Firstly, a retrospective study with relatively small number of patients might cause selection bias. To generalize the results of the study, a large RCT is preferred. Secondly, there were several differences between the groups. To adjust for these differences, we used multivariate analysis by Cox proportional hazards model. However, there was a possibility that it may not be enough to correct these differences. Finally, there were possibilities that we could not collect all composite events. We collected the data of VTE recurrence and bleeding events via the electronic medical records in Asan Medical Center. Additionally, we used insurance data for patient mortality. However, we could not get data of VTE recurrence and bleeding events that were not electronically recorded.

\section{CONCLUSIONS}

The present study shows that rivaroxaban is safe and efficacious for the treatment of VTE in patients with urologic cancer compared with dalteparin. In addition, rivaroxaban shows less HRs than dalteparin. Thus, DOACs can be regarded as a valuable option in treating urologic cancer-associated VTE. Further prospective studies with more patients are warranted to consolidate the safety and efficacy of rivaroxaban.

\section{CONFLICT OF INTEREST}

The authors claim no conflicts of interest.

\section{REFERENCES}

1. Franchini M, Bonfanti C, Lippi G. Cancer-associated thrombosis: investigating the role of new oral anticoagulants. Thromb Res 2015;135:777-81.

2. Vedovati MC, Giustozzi M, Becattini C. Venous thromboembolism and cancer: Current and future role of di- rect-acting oral anticoagulants. Thromb Res 2019;177:3341.

3. Scarpa RM, Carrieri G, Gussoni G, Tubaro A, Conti G, Pagliarulo V, et al. Clinically overt venous thromboembolism after urologic cancer surgery: results from the @RISTOS Study. Eur Urol 2007;51:130-5.

4. Zareba P, Duivenvoorden WCM, Pinthus JH. Thromboembolism in Patients with Bladder Cancer: Incidence, Risk Factors and Prevention. Bladder Cancer 2018;4:139-47.

5. Fernandes CJ, Morinaga LTK, Alves JL Jr, Castro MA, Calderaro D, Jardim CVP, et al. Cancer-associated thrombosis: the when, how and why. Eur Respir Rev 2019 Mar 27;28(151). pii: 180119. https://doi.org/10.1183/16000617. 0119-2018.

6. Lee AY, Levine MN, Baker RI, Bowden C, Kakkar AK, Prins $\mathrm{M}$, et al. Low-molecular-weight heparin versus a coumarin for the prevention of recurrent venous thromboembolism in patients with cancer. $\mathrm{N}$ Engl $\mathrm{J}$ Med 2003;349:146-53.

7. Smrke A, Gross PL. Cancer-Associated Venous Thromboembolism: A Practical Review Beyond Low-MolecularWeight Heparins. Front Med (Lausanne) 2017;4:142.

8. Cohen AT, Hamilton M, Mitchell SA, Phatak H, Liu X, Bird A, et al. Comparison of the Novel Oral Anticoagulants Apixaban, Dabigatran, Edoxaban, and Rivaroxaban in the Initial and Long-Term Treatment and Prevention of Venous Thromboembolism: Systematic Review and Network Meta-Analysis. PLoS One 2015;10:e0144856.

9. Kearon C, Akl EA, Ornelas J, Blaivas A, Jimenez D, Bounameaux $\mathrm{H}$, et al. Antithrombotic Therapy for VTE Disease: CHEST Guideline and Expert Panel Report. Chest 2016;149:315-52.

10. Uppuluri EM, Burke KR, Haaf CM, Shapiro NL. Assessment of venous thromboembolism treatment in patients with cancer on low molecular weight heparin, warfarin, and the direct oral anticoagulants. J Oncol Pharm Pract 2019;25:261-8.

11. Brodie MM, Newman JC, Smith T, Rockey DC. Severity of Gastrointestinal Bleeding in Patients Treated with Direct-Acting Oral Anticoagulants. Am J Med 2018;131: 573.e9-573.e15.

12. Sherwood MW, Nessel CC, Hellkamp AS, Mahaffey KW, Piccini JP, Suh EY, et al. Gastrointestinal Bleeding in Patients With Atrial Fibrillation Treated With Rivaroxaban or Warfarin: ROCKET AF Trial. J Am Coll Cardiol 2015; 66:2271-81

13. Cheung KS, Leung WK. Gastrointestinal bleeding in patients on novel oral anticoagulants: Risk, prevention and management. World J Gastroenterol 2017;23:1954-63.

14. Wallis CJD, Juvet $\mathrm{T}$, Lee $\mathrm{Y}$, Matta R, Herschorn S, Kodama R, et al. Association Between Use of Antithrombotic Medication and Hematuria-Related Complications. 
JAMA 2017;318:1260-71.

15. Streiff MB, Holmstrom B, Angelini D, Ashrani A, Bockenstedt PL, Chesney C, et al. NCCN Guidelines Insights: Cancer-Associated Venous Thromboembolic Disease, Version 2.2018. J Natl Compr Canc Netw 2018; 16:1289-303.

16. Khorana AA, Noble S, Lee AYY, Soff G, Meyer G, O'Connell $\mathrm{C}$, et al. Role of direct oral anticoagulants in the treatment of cancer-associated venous thromboembolism: guidance from the SSC of the ISTH. J Thromb Haemost 2018;16:1891-4.

17. Raskob GE, van Es N, Verhamme P, Carrier M, Di Nisio M, Garcia D, et al. Edoxaban for the Treatment of Cancer-Associated Venous Thromboembolism. N Engl J Med 2018;378:615-24.

18. Young AM, Marshall A, Thirlwall J, Chapman O, Lokare A, Hill C, et al. Comparison of an Oral Factor Xa Inhibitor With Low Molecular Weight Heparin in Patients With Cancer With Venous Thromboembolism: Results of a Randomized Trial (SELECT-D). J Clin Oncol 2018;36: 2017-23.

19. Kim SA, Yhim HY, Bang SM. Current Management of Cancer-associated Venous Thromboembolism: Focus on Direct Oral Anticoagulants. J Korean Med Sci 2019;34: e52.

20. Dong Y, Wang Y, Ma RL, Liu M, Gao JZ, Su WY, et al. Efficacy and safety of direct oral anticoagulants versus low-molecular-weight heparin in patients with cancer: a systematic review and meta-analysis. J Thromb Thrombolysis 2019;48:400-12.

21. Short NJ, Connors JM. New oral anticoagulants and the cancer patient. Oncologist 2014;19:82-93.

22. EINSTEIN Investigators, Bauersachs R, Berkowitz SD, Brenner B, Buller HR, Decousus H, et al. Oral rivaroxaban for symptomatic venous thromboembolism. N Engl J Med
2010;363:2499-510.

23. Lee JH, Oh YM, Lee SD, Lee JS. Rivaroxaban versus Low-Molecular-Weight Heparin for Venous Thromboembolism in Gastrointestinal and Pancreatobiliary Cancer. J Korean Med Sci 2019;34:e160.

24. Lee JH, Hyun DG, Choi CM, Lee JC, Kim WS, Oh YM, et al. A Retrospective Study on Efficacy and Safety of Rivaroxaban and Dalteparin for Long-Term Treatment of Venous Thromboembolism in Patients with Lung Cancer. Respiration 2019;98:203-11.

25. Lee JH, Lee JH, Jo KW, Huh JW, Oh YM, Lee JS. Comparison of rivaroxaban and dalteparin for the long-term treatment of venous thromboembolism in patients with gynecologic cancers. J Gynecol Oncol 2019 Aug 5. doi: 10.3802/jgo.2020.31.e10. [Epub]

26. Chan NC, Eikelboom JW, Weitz JI. Evolving Treatments for Arterial and Venous Thrombosis: Role of the Direct Oral Anticoagulants. Circ Res 2016;118:1409-24.

27. Li A, Garcia DA, Lyman GH, Carrier M. Direct oral anticoagulant (DOAC) versus low-molecular-weight heparin (LMWH) for treatment of cancer associated thrombosis (CAT): A systematic review and meta-analysis. Thromb Res 2019;173:158-63.

28. Weinz C, Schwarz T, Kubitza D, Mueck W, Lang D. Metabolism and excretion of rivaroxaban, an oral, direct factor Xa inhibitor, in rats, dogs, and humans. Drug Metab Dispos 2009;37:1056-64.

29. Desai J, Kolb JM, Weitz JI, Aisenberg J. Gastrointestinal bleeding with the new oral anticoagulants--defining the issues and the management strategies. Thromb Haemost 2013;110:205-12.

30. Frydman A. Low-molecular-weight heparins: an overview of their pharmacodynamics, pharmacokinetics and metabolism in humans. Haemostasis 1996;26 Suppl 2:24-38. 